\title{
Synthesis of meso- tetrakis (2-chloroquinolin-3-yl) porphyrins
}

\author{
Mandha Amaravathi*, Maradolla Mohan Babu, and Garimella Chandramouli \\ Department of Chemistry, National Institute of Technology Warangal (AP)-506004, India \\ E-mail: ammi_reddy@hotmail.com,mohanorg@yahoo.com
}

\begin{abstract}
meso-Tetrakis (-2-chloroquinolin-3-yl) porphyrins were synthesized from 2-chloroquinoline-3carboxaldehydes and pyrrole in $1: 1$ ratio in propionic acid at $140{ }^{\circ} \mathrm{C}$ for 4 hours. Different substituted 2-chloroquinoline-3-carboxaldehydes were synthesized from corresponding $\mathrm{N}$-aryl acetamides by Vilsmeier-Haack cyclization. The aldehydes were obtained in better yields, when acetamides, dimethyl formamide and $\mathrm{POCl}_{3}$ were taken in 1:3: 7 molar ratios at $70-75{ }^{\circ} \mathrm{C}$
\end{abstract}

Keywords: N-aryl acetamides, Vilsmeier-Haack cyclization, 2-chloroquinoline-3carboxaldehydes, meso-Tetrakis(-2-chloroquinoline-3-yl) porphyrins

\section{Introduction}

There is a particular interest for synthesis of porphyrins bearing heterocyclic moieties due to varied biological $^{1}$, photophysical ${ }^{2}$ and electronic properties ${ }^{3}$. The heteroaryl porphyrin with diverse substitutents is important for the studies of biomimetic and molecular recognition properties $^{4,5}$. A good number of porphyrins containing nitrogen heterocycles have been prepared and their properties were studied. Substituted nitrogen heterocyclic porphyrins are of particular interest $^{6}$ as they provide sites for metal coordination, hydrogen bonding, alkylation and modulating electronic properties. Indeed, pyridine substituents have yielded a broad array of metal coordinated multi porphyrin architectures ${ }^{7}$ and imidazole groups have yielded stacked multi porphyrin assembles ${ }^{8}$. Pyrimidine ${ }^{9}$, purine $^{10}$ or pyrazole $^{11}$ units have enabled molecular recognition and self-assembly studies of porphyrins with complementary molecules. Several quinoline derivatives have been found to possess useful biological activities such as bactericidal $^{12}$, antitumor ${ }^{13}$, antimalarial ${ }^{14}$, antinflamatory ${ }^{15}$. The benzo and hetero fused quinolines are known to bind to DNA topoisomerase and display cytotoxic and antitumor activities $^{16}$. [5,15-Bis(8-quinolyl)porphyrinato]zincII has been used for molecular recognition of carbohydrates ${ }^{17}$. The above applications of the meso-substituted heterocyclic porphyrins inspired us to synthesize the new meso-tetrakis(2-chloroquinolin-3-yl)porphyrins (2a-2d). Porphyrins themselves are having high applications in organic chemistry, so by substituting quinoline 
moiety in meso position of porphyrins, it may possess high potential towards the biological applications.

\section{Results and Discussion}

2-Chloroquinoline-3-carboxaldehydes (1a-1d) were synthesized starting from $\mathrm{N}$-aryl acetamides by Vilsmeier-Haack cyclization ${ }^{18}$. Several experiments were conducted to produce these aldehydes in better yields by changing the ratios of $\mathrm{N}$-aryl acetamide, $\mathrm{POCl}_{3}$ and dimethyl formamide at different temperatures. 2-Chloroquinoline-3-carboxaldehydes (1a-1d) were obtained in higher yields at $70-75^{\circ} \mathrm{C}$ using $\mathrm{N}$ - aryl acetamides, DMF and $\mathrm{POCl}_{3}$ in 1:3: 7 molar ratios.

meso-Tetrakis(-2-chloroquinolin-3-yl) porphyrins (2a-2d) were synthesized in 10-12\% yield by treating the 2-chloroquinoline-3-carboxaldehydes (1a-1d) with pyrrole in 1:1 ratio in propionic acid at $140{ }^{\circ} \mathrm{C}$ for 4 hours (Scheme 1). When the reaction was conducted according to the Lindsey procedure (TFA / DCM), the porphyrin obtained was in negligible quantity ${ }^{19}$, where as by using more propionic acid ( $25 \mathrm{ml} / 1$ millimole of aldehyde), the yield of porphyrin got increased. The porphyrins (2a-2d) were purified by passing through a flash-column using a mixture of chloroform and methanol $(98: 2)$ as eluent.

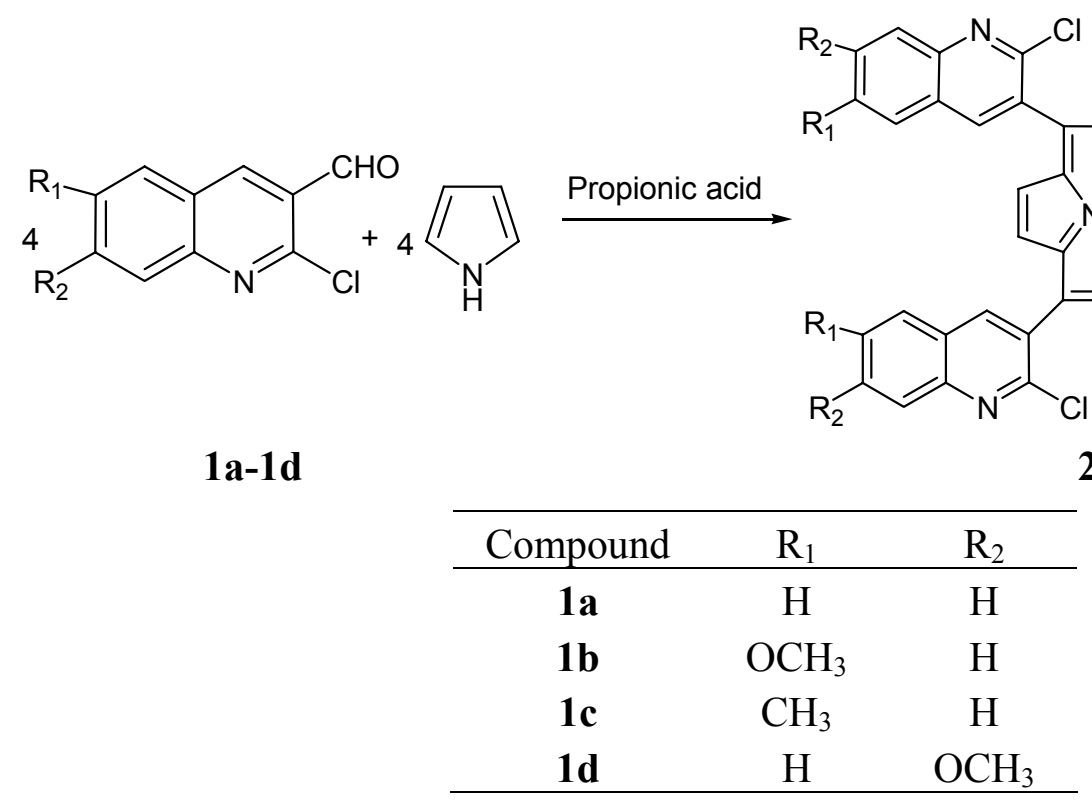

\section{Scheme 1}

meso-Tetrakis(-2-chloroquinolin-3-yl) porphyrins (2a-2d) exhibit the phenomenon of atropisomerism, a typical feature of meso- (2-substituted-aryl)porphyrins ${ }^{20}$. This is due to a high rotational energy barrier caused by steric hindrance between the quinoline 2-chloro group and 
the porphyrin $\beta$-protons. Four distinct bands were observed by TLC for porphyrins 2a-2d. Attempts were made to separate these atropisomers by column chromatography (flash silica gel 230-400 mesh). TLC studies indicate that each one of the individual isomeric fractions of $\mathbf{2 a - 2 d ,}$ becomes a mixture of atropisomers, on standing at ambient temperature. The ${ }^{1} \mathrm{H}$ NMR spectra show a complex resonances for the central porphyrin N-H and $\beta$-protons for porphyrins $\mathbf{2 a - 2 d . ~}$ Where as, the $\mathrm{C}_{4}$ proton of the quinoline appears as a singlet around $\delta 8.60 \mathrm{ppm}$ in all the porphyrins. UV-Vis spectra of quinoline porphyrins were recorded at $1 \times 10^{-5}$ mol concentrations in $\mathrm{CHCl}_{3}$. Highly characteristic spectra were obtained for quinoline porphyrins 2a-d, in which the $\mathrm{B}$ band is prominent at $430 \mathrm{~nm}$ and Q bands are observed at 520, 600 and $661 \mathrm{~nm}$. The IR spectra contain a characteristic macrocyclic bending frequency of $970 \mathrm{~cm}^{-1}$ for $\mathbf{2 a - 2 d}$.

\section{Experimental Section}

General Procedures. UV-vis spectra were recorded on a SHIMADZU UV 160 A UV-vis-NIR spectrophotometer, using chloroform as solvent. IR spectra were recorded as $\mathrm{KBr}$ pellets using a SHIMADZU 8010 FTIR spectrophotometer. ${ }^{1} \mathrm{H}$ NMR spectra were recorded on a VARIAN FT $500 \mathrm{MHz}$ instrument using $\mathrm{CDCl}_{3}$ and DMSO- $\mathrm{d}_{6}$ as solvent and TMS as internal reference. FAB- Mass spectra were recorded on a VG Micromass $7070 \mathrm{H}(\mathrm{F}$, or $\mathrm{CI})$ auto spectrometer. The $\mathrm{C}, \mathrm{H}, \mathrm{N}$ analysis of the compounds was performed on a Carlo Erba Model EA $1108 \mathrm{CHNS}-\mathrm{O}$ elemental analyzer. Porphyrins were purified by flash column chromatography using 230-400 mesh silica gel (Aldrich).

Synthesis of meso- tetrakis(2-chloroquinolin-3-yl)porphyrin (2a). 2-Chloroquinoline-3carboxaldehyde (1a, $383 \mathrm{mg}, 2 \mathrm{mmol}$ ) was dissolved in $50 \mathrm{ml}$ of freshly distilled propionic acid and pyrrole (134 mg, $2 \mathrm{mmol}$ ) was added. The reaction mixture was stirred at $140{ }^{\circ} \mathrm{C}$ in open air for $4 \mathrm{~h}$. Then the propionic acid was distilled off under reduced pressure and the residue was cooled to room temperature. $30 \mathrm{ml}$ of cold water was added to the residue and neutralized carefully with saturated sodium bicarbonate solution. The dark colored residue was filtered and dried. The crude product was purified by flash column chromatography using chloroform: methanol (95: 5) mixture as eluent. Four separate atropisomeric fractions were collected, which after concentration gave a dark purple solid $\mathbf{2 a}(58 \mathrm{mg}, 12.1 \%)$. Identification and characterization of all the four fractions indicated that they contain the same compound. The first fraction was isolated as a single isomer. $\mathrm{Mp}:>300{ }^{\circ} \mathrm{C}$. Anal. calcd. for $\mathrm{C}_{56} \mathrm{H}_{30} \mathrm{~N}_{8} \mathrm{Cl}_{4}: \mathrm{C}, 70.38$; H, 3.16; N, 11.71. Found: C, 70.25; H, 3.22; N, 11.73\%. FAB-MS m/z $957(\mathrm{M}+1)^{+}$requires 956. UV: $\lambda_{\max } \mathrm{nm}\left(\mathrm{CHCl}_{3}\right)(\log \xi): 430$ (5.504), 520(4.53), 601 (4.30), 660 (4.00). ${ }^{1} \mathrm{H}$ NMR $\left(\mathrm{CDCl}_{3}+\mathrm{DMSO}-\mathrm{d}_{6}\right) \delta$ ppm: $9.05(\mathrm{~m}, 8 \mathrm{H}$, pyrrole $\beta$-protons $), 8.67$ (s, 4H, quinoline $\left.\mathrm{C}_{4}-\underline{\mathrm{H}}\right), 8.07$ (m, 8H, quinoline $\left.\mathrm{C}_{5} \& \mathrm{C}_{8}-\underline{\mathrm{H}}\right), 7.82\left(\mathrm{~m}, 4 \mathrm{H}\right.$, quinoline $\left.\mathrm{C}_{6}-\underline{\mathrm{H}}\right), 7.60\left(\mathrm{~m}, 4 \mathrm{H}\right.$, quinoline $\left.\mathrm{C}_{7}-\underline{\mathrm{H}}\right),-2.63$ (s, 2H, porphyrin $\mathrm{N}-\underline{\mathrm{H}})$. IR (KBr) cm $\mathrm{cm}^{-1}: 3436$ (w, porphyrin $\mathrm{N}-\mathrm{H}$ stretch), 1570, 1458, 1420, 1362 
(m, aromatic $\mathrm{C}=\mathrm{C}, \mathrm{C}=\mathrm{N}$ ring stretch), 973, 956 (m, porphyrin macrocyclic bend), 801, 785, 758 (s, aromatic $\mathrm{C}=\mathrm{C}-\mathrm{H}$ out-of plane bend).

Synthesis of meso- tetrakis(2-chloro-6-methoxyquinolin-3-yl)porphyrin (2b). A mixture of $443 \mathrm{mg}$ ( $2 \mathrm{mmol}$ ) of 2-chloro-6-methoxyquinoline-3-carboxaldehyde (1b) and $134 \mathrm{mg}$ (2 mmol) of freshly distilled pyrrole was dissolved in $50 \mathrm{ml}$ of propionic acid and heated at $140^{\circ} \mathrm{c}$ for $4 \mathrm{hrs}$ with stirring. After the usual workup procedure was conducted as described above, the black residue obtained was subjected to column chromatography on flash silica gel using chloroform : methanol (98:2) mixture as eluent. Four separate purple colored atropisomeric fractions were collected, which after concentration gave a dark purple solid $\mathbf{2 b}$ (62 $\mathrm{mg}, 11.5 \%)$. It was observed that all the four fractions contain the same compound. The first fraction was isolated as a single isomer. $\mathrm{Mp}$ : $>300{ }^{\circ} \mathrm{C}$ Anal. calcd. for $\mathrm{C}_{60} \mathrm{H}_{38} \mathrm{~N}_{8} \mathrm{O}_{4} \mathrm{Cl}_{4}: \mathrm{C}, 66.92 ; \mathrm{H}, 3.56 ; \mathrm{N}, 10.41$. Found: C, 66.86; H, 3.62; N, 10.50\%: FAB-MS m/z $1077(\mathrm{M}+1)^{+}$, requires 1076 . UV: $\lambda_{\max } \mathrm{nm}\left(\mathrm{CHCl}_{3}\right)$ $(\log \xi): 430$ (5.53), 520(4.84), 600 (4.31), 661 (4.00). ${ }^{1} \mathrm{H}$ NMR ( $\left.\mathrm{CDCl}_{3}+\mathrm{DMSO}_{6}\right) \delta \mathrm{ppm}$ : $9.06\left(\mathrm{~m}, 8 \mathrm{H}\right.$, pyrrole $\beta$-protons), $8.66\left(\mathrm{~s}, 4 \mathrm{H}\right.$, quinoline $\left.\mathrm{C}_{4}-\underline{\mathrm{H}}\right), 7,94\left(\mathrm{~d}, 4 \mathrm{H}\right.$, quinoline $\left.\mathrm{C}_{8}-\underline{\mathrm{H}}\right), 7.52$ (d, 4H, quinoline $\left.\mathrm{C}_{7}-\underline{\mathrm{H}}\right), 7.18\left(\mathrm{~s}, 4 \mathrm{H}\right.$, quinoline $\left.\mathrm{C}_{5}-\underline{\mathrm{H}}\right), 3.96\left(\mathrm{~s}, 12 \mathrm{H}, 4 \times \mathrm{O}-\mathrm{C}_{3}\right),-2.60(\mathrm{~s}, 2 \mathrm{H}$, porphyrin $\mathrm{N}-\underline{\mathrm{H}})$. IR (KBr) $\mathrm{cm}^{-1}: 3426$ (w, porphyrin $\mathrm{N}-\mathrm{H}$ stetch), 1622, 1588, 1494, 1462 (m, aromatic $\mathrm{C}=\mathrm{C}, \mathrm{C}=\mathrm{N}$ ring stretch), 969, 959 (m, porphyrin macrocyclic bend), 831,797 (s, aromatic $\mathrm{C}=\mathrm{C}-\mathrm{H}$ out-of plane bend).

Synthesis of meso- tetrakis(2-chloro-6-methylquinolin-3-yl)porphyrin (2c). A mixture of 410 $\mathrm{mg}(2 \mathrm{mmol})$ of 2-chloro-6-methylquinoline-3-carboxaldehyde (1c) and $134 \mathrm{mg}$ (2 mmol) of freshly distilled pyrrole was dissolved in $50 \mathrm{ml}$ of propionic acid and heated at $140^{\circ} \mathrm{C}$ for $4 \mathrm{hrs}$ with stirring. After the usual work up procedure was conducted as described above, the black residue obtained was subjected to column chromatography on flash silica gel using chloroform : methanol (97:3) mixture as eluent. Four separate purple colored atropisomeric fractions were collected, which after concentration gave a dark purple solid 2c (54 mg, 10.6\%). Identification and characterization of all the four fractions indicated that they contain the same compound. The first fraction was isolated as a single isomer. $\mathrm{Mp}:>300{ }^{\circ} \mathrm{C}$. Anal. calcd. for $\mathrm{C}_{60} \mathrm{H}_{38} \mathrm{~N}_{8} \mathrm{Cl}_{4}: \mathrm{C}$, 71.15; H, 3.78; N, 11.06. Found: C, 71.02; H, 3.86; N, 11.12\%. FAB-MS m/z $1013(\mathrm{M}+1)^{+}$, requires 1012. $\mathrm{UV}: \lambda_{\max } \mathrm{nm}\left(\mathrm{CHCl}_{3}\right)(\log \xi): 430$ (5.566), 520(4.64), $601(4.11), 661(4.00) .{ }^{1} \mathrm{H}$ NMR $\left(\mathrm{CDCl}_{3}+\mathrm{DMSO}_{-} \mathrm{d}_{6}\right) \delta \mathrm{ppm}: 9.07$ (m, 8H, pyrrole $\beta$-protons), 8.56 (s, 4H, quinoline $\mathrm{C}_{4^{-}}$ $\underline{\mathrm{H}}), 7,95\left(\mathrm{~d}, 4 \mathrm{H}\right.$, quinoline $\left.\mathrm{C}_{8}-\underline{\mathrm{H}}\right), 7.65\left(\mathrm{~m}, 8 \mathrm{H}\right.$, quinoline $\left.\mathrm{C}_{5} \& \mathrm{C}_{7}-\underline{\mathrm{H}}\right), 2.56\left(\mathrm{~s}, 12 \mathrm{H}, 4 \mathrm{x}-\underline{\mathrm{C}}_{3}\right)$, $2.60(\mathrm{~s}, 2 \mathrm{H}$, porphyrin $\mathrm{N}-\underline{\mathrm{H}})$. IR (KBr) cm $\mathrm{cm}^{-1}: 3432$ (w, porphyrin N-H stretch), 1602, 1578, 1484, $1452(\mathrm{~m}$, aromatic $\mathrm{C}=\mathrm{C}, \mathrm{C}=\mathrm{N}$ ring stretch), 970, 956 (m, porphyrin macrocyclic bend), 812, 786 ( $\mathrm{s}$, aromatic $\mathrm{C}=\mathrm{C}-\mathrm{H}$ out-of plane bend).

Synthesis of meso- tetrakis(2-chloro-7-methoxyquinolin-3-yl)porphyrin (2d). A mixture of $443 \mathrm{mg}(2 \mathrm{mmol})$ of 2-chloro-7-methoxyquinoline-3-carboxaldehyde (1d) and $134 \mathrm{mg}$ (2 mmol) of freshly distilled pyrrole was dissolved in $50 \mathrm{ml}$ of propionic acid and heated at $140{ }^{\circ} \mathrm{C}$ for $4 \mathrm{~h}$ while stirring. Following the usual workup procedure as described above, the black residue procured was subjected to column chromatography on flash silica gel using chloroform : 
methanol (98:2) mixture as eluent. Four separate purple colored atropisomeric fractions were collected, which after concentration gave a dark purple solid $2 \mathbf{d}(62 \mathrm{mg}, 11.5 \%)$. All the four fractions obtained contain the same compound. The first fraction was isolated as a single isomer. $\mathrm{Mp}:>300{ }^{\circ} \mathrm{C}$.Anal. calcd. for $\mathrm{C}_{60} \mathrm{H}_{38} \mathrm{~N}_{8} \mathrm{O}_{4} \mathrm{Cl}_{4}$ : C, 66.92; H, 3.56; N, 10.41. Found: C, 66.98; $\mathrm{H}, 3.64 ; \mathrm{N}, 10.35 \%$. FAB-MS m/z $1077(\mathrm{M}+1)^{+}$, requires 1076. UV: $\lambda_{\max } \mathrm{nm}\left(\mathrm{CHCl}_{3}\right)(\log \xi)$ : 430 (5.50), 520(4.41), 601 (4.21), 661 (4.00). ${ }^{1} \mathrm{H} \mathrm{NMR}\left(\mathrm{CDCl}_{3}+\mathrm{DMSO}-\mathrm{d}_{6}\right) \delta \mathrm{ppm}: 9.05(\mathrm{~m}$, $8 \mathrm{H}$, pyrrole $\beta$-protons), 8.63 (s, $4 \mathrm{H}$, quinoline $\left.\mathrm{C}_{4}-\underline{\mathrm{H}}\right), 7,84\left(\mathrm{~d}, 4 \mathrm{H}\right.$, quinoline $\left.\mathrm{C}_{5}-\underline{\mathrm{H}}\right), 7 . .37$ (s, 4H, quinoline $\left.\mathrm{C}_{8}-\underline{\mathrm{H}}\right), 7.27\left(\mathrm{~d}, 4 \mathrm{H}\right.$, quinoline $\left.\mathrm{C}_{6}-\underline{\mathrm{H}}\right), 3.98\left(\mathrm{~s}, 12 \mathrm{H}, 4 \mathrm{x} \mathrm{O}-\mathrm{C}_{3}\right),-2.60$ (s, 2H, porphyrin $\mathrm{N}-\underline{\mathrm{H}}$ ). IR (KBr) cm $\mathrm{cm}^{-1}: 3430$ (w, pophyrin N-H stretch), 1620, 1585, 1492, 1464 (m, aromatic $\mathrm{C}=\mathrm{C}, \mathrm{C}=\mathrm{N}$ ring stretch), 968, 958 (m, porphyrin macrocyclic bend), 832, 798 (s, aromatic $\mathrm{C}=\mathrm{C}$ $\mathrm{H}$ out-of plane bend).

\section{Conclusions}

meso-Tetrakis (-2-chloroquinolin-3-yl) porphyrins (2a-2d) were obtained in 10-12\% yields when propionoic acid is used in proportions of $25-30 \mathrm{ml}$ for $1 \mathrm{mmol}$ of quinoline aldehyde. These porphyrins may be useful for molecular recognition of carbohydrates.

\section{Acknowledgements}

One of the authors (M. Amaravathi) gratefully acknowledges the Department of Science and Technology, New Delhi, INDIA, for sanctioning Women Scientist Scheme-A. We also thank to the National Institute of Technology Warangal India for providing lab facilities.

\section{References}

1. Collman, J. P.; Wang, Z. Chemtracts-Org.Chem. 1999, 12, 229.

2. Piotrowiak, P. Chem. Soc. Rev. 1999, 28, 143.

3. Yang, S. I.; Seth, J.; Strachan, J.-P.; Gentenann, S.; Kim, D.; Holten, D.; Lindsey, J. S.; Bocian, D. F. J. Phorphyrins Phthalocyanines. 1999, 3, 117.

4. Lindsey, J. S. In Metalloporphyrin - Catalysed Oxidations Montanari, F.; Casella, L.; Eds. Kluwar Academic Publications: The Netherlands, 1994, 49.

5. Lindsey, J. S. In The Porphyrin Handbook Kandish, K. M,; Smith, K. M.; Guilard, R. Eds. Academic Press: San Diego, C.A. 2000, 1, 45.

6. Chamborn, J.-C.; Heitz, V.; Sauvage, J.-P. In The Porphyrin Handbook, Kadish, K. M.; Smith, K. M.; Guilard, R. Eds.; Academic Press: San Diego, CA, 2000, Vol. 6, pp 1-42 
7. (a) Ding, L.; Casas, C.; Etemad- Moghadam, G.; Meunier, B.; Cros, S. New J. Chem. 1990, 14, 421. (b) Sari, M. A.; Battioni, J. P.; Dupre, D.; Mansuy, D.; Le Pecq, J. B. Biochemistry. 1990, 29, 4205. (c) Milgrom, L. R.; Hill, J. P.; Dempsey, P. J. F. Tetrahedron. 1994, 50, 13477. (d) Takeuchi, M.; Imada, T.; Ikeda, M.; Shinkai, S. Tetrahedron Lett. 1998, 39, 7897.

8. (a) Milgrom, L. R.; Dempsey, P. J. F.; Yahioglu,G. Tetrahedron. 1996, 52, 9877. (b) Kobuke, Y.; Miyaji, H. Bull. Chem. Soc. Jpn. 1996, 69, 3563.

9. (a) Sessler, J. L.; Wang, B.; Harriman. A. J. Am. Chem. Soc. 1995, 117, 704. (b) Maes, W.; Vanderhaeghen, J.; Smeets, S.; Asokan, C. V.; Lieven, M. V. R.; (c) Filip, E. D. P.; Smet, M.; Dehaen W. J. Org. Chem. 2006, 71, 2987. (c) Maes, W.; Dehaen, W. Synlett 2003, 1, 79. (d) Smeets, S.; Asokan, C. V.; Motrnans, F.; Dehaen, W. J. Org. Chem. 2000, 65, 5882. (e) Motmans, F.; Ceulemans, E.; Smeets, S.; Dehaen, W. Tetrahedron Lett. 1999, 40, 7545.

10. Drain, C. M.; Fisher, R.; Nolen, E. G.; Lehn, J. M. J. Chem. Soc., Chem. Commun. 1993, 245.

11 (a) Ikeda, C.; Nagahara, N.; Motegi, E.; Yoshioka, N.; Inoue, H. Chem. Commun. 1999, 1759. (b)Bruix, M.; Elguero, J.; Meutermans, W. J. Chem. Res. 1992, 370. (c) Guo, C. C.; Ren, T. G.; Wang, J.; Li, C. Y.; Sond, J. X. J. Porphyrins Phthalocyanines. 2005, 9, 430.

12 Patel, H. V.; Vyas, K. V.; Fernandes, P. S. Indian. J. Chem. 1990, 29(B), 836.

13 Sukhova, N. M.; Lidak, M.; Zidermane, A.; Pelevina, I.; Voronia, S. S. Khim-Farm. Zh. 1989, 23, 1226.

14 Craig, J. C.; Person, P. E. J. Med. Chem. 1971, 14, 1221.

15 Dillard, R. D.; Pavey, D. E.; Benslay, D. N. J. Med. Chem. 1973, 16, 251.

16 (a) Spieer, J. A.; Gamage, S. A.; Atwell, G. J.; Fnlay, G. J.; Bagulay, B. C.; Denny, W. A. J. Med.Chem. 1977, 40, 1919. (b) Lyon, M. A.; Lawrence, S.; William D. J.; Jackson, Y. A. J. Chem. Soc., Perkin Trans. 1, 1999, 437.

17 (a) Mizutani, T.; Kurahashi. T.; Murakami. T.; Matsumi, N.; Ogoshi, H. J. Am. Chem. Soc. 1997, 119, 8991. (b) McCurry, J.; Roberts, J. E. Polyhedron. 1990, 9, 2527.

18 (a) Meth-Cohn, O.; Narine, B.; Tarnowski, B. J chem. Soc., Perkin Trans. 1, 1981, 1, 1520.

(b)Ali, M. M.; Tasneem,; Rajanna, K. C.; Sai Prakash, P. K. Synlett. 2001, 2, 251.

19 Lindsey, J. S.; Schrieman, I. C.; Hus, H. C.; Kearney, P. C.; Marguerettaz, A. M. J. Org. Chem. 1987, 52, 827.

20 Crossley, M. J.; Field, L. D.; Forster, A. J.; Harding, M. M,; Sternhell, S. J. Am. Chem. Soc. 1987, 109, 341. 